\title{
A METHOD FOR SUMMABILITY OF LAGRANGE INTERPOLATION
}

\section{DETLEF H. MACHE}

Iniversität Dortmund, Lehrstuhl Mathematik VIII

Postfach 5005 00. D - 4600 Dortmund 50

Germany

(Received August 27, 1992 and in revised form December 8, 1992)

ABSTRACT. The author uses in this paper a technique from numerical integration (see [9]) to get a discretely defined operator, which is a modification of the Lagrange operator. Therefore we improve with the linear summation method $L_{n}^{\star}$ a result presented in [12] and we also point out a solution for a problem of P.L.Butzer in the proceedings of the Budapest conference in 1980.

KEY WORDS AND PHRASES. Approximation by positive linear operators, discrete linear operators, Lagrange Interpolation, pointwise estimates, second order modulus of continuity, Lipschitz - type maximalfunction, Butzer's problem.

1992 AMS SUBJECT CLASSIFICATION CODES. 41A10, 41 A25, 41A36.

\section{INTRODUCTION.}

Let $\Pi_{n}$ be the set of algebraic polynomials of degree $\leq n$ and let $M$ be the triangular matrix of points

$$
M=\left\{\begin{array}{cccccc}
z_{0,0} & & & & \\
z_{0,1} & z_{1,1} & & & & \\
\vdots & \vdots & & & \\
z_{0, n} & z_{1, n} & \cdots & z_{n, n} & \\
\vdots & \vdots & & \vdots & \ddots
\end{array}\right.
$$

where

$$
1 \geq z_{0, n}>z_{1, n}>\cdots>z_{n, n} \geq-1
$$

For any continuous function $f \in C(I)$ with $I:=[-1,1]$ we define the $n$ - th Lagrange interpolation polynomial $L_{n} f$ with respect to the set of points $z_{k, n}=\cos \frac{2 k+1}{2(n+1)} \pi, k=0,1, \cdots, n, n \in \mathbb{N}_{0}$, to be that polynomial of degree $\leq n$, which interpolates the values $\left(z_{k, n}, f\left(z_{k, n}\right)\right)$.

Therefore the $(n+1)$ points $z_{k, n}$ are the zeros of the $(n+1)$ - th Chebyshev polynomial $T_{n+1}(x)=$ $\cos ((n+1) \arccos x)$. These Lagrange operators $L_{n}: C(I) \rightarrow \Pi_{n}, n \in \mathbb{N}_{0}$, are given by

$$
L_{n}\left(z_{0, n}, \cdots, z_{n, n} ; f, x\right)=\sum_{k=0}^{n} f\left(z_{k, n}\right) \frac{T_{n+1}(x)}{\left(x-z_{k, n}\right) T_{n+1}{ }^{\prime}\left(z_{k, n}\right)} .
$$

If we define $\omega_{0}=\frac{1}{\pi}$ and $\omega_{\jmath}=\frac{2}{\pi}, j \geq 1$, one can easily see that 


$$
\frac{T_{n+1}(. r)}{\left(. r-z_{\ell, n}\right) T_{n+1}^{\prime}\left(z_{\ell, n}\right)}=\frac{\pi}{n+1} \sum_{\jmath=0}^{n} \omega_{j} T_{\jmath}(. x) T_{\jmath}\left(z_{k, n}\right) .
$$

We use for $f . g: I \rightarrow \mathbb{R}$ the inner product

$$
[f \cdot g]_{n}=\frac{\pi}{n+1} \sum_{h=0}^{n} f(z h, n) g(z h, n) .
$$

and so one finds the representation

$$
L_{n}\left(z_{0, n}, \cdots, z_{n, n}: f, x\right)=\sum_{j=0}^{n} \omega_{j}\left[f, T_{\jmath}\right]_{n} T_{\jmath}(x) .
$$

It is known about the sequence $L=\left(L_{n}\right)$ that it does not converge pointwise to the identity on the space $C^{\prime}(I)$.

This negative behaviour was the impulse for some authors to find and to investigate approximation methods in a form of a fine modification, which have better properties.

For instance in this paper we consider linear approximation operators $\hat{L}_{n}: C(I) \rightarrow \Pi_{n}, n \in \mathbb{N}$, given by

$$
\left(\hat{L}_{n} f\right)(x)=\sum_{\jmath=0}^{n} \hat{\beta}_{\jmath, n} \omega_{\jmath}\left[f, T_{\jmath}\right]_{n} T_{\jmath}(x) .
$$

An open problem is to find the "convergence - multipliers" $\hat{\beta}_{3, n}$ such that, with respect to the uniform norm, we have for all $f \in C(I)$

$$
\lim _{n \rightarrow \infty}\left\|f-\hat{L}_{n} f\right\|=0 .
$$

Let us use the well - known translation operator $\tau_{x}: X \rightarrow X, x \in I$, defined by (see [2], [3])

$$
\left(\tau_{x} f\right)(t)=\frac{1}{2}\left[f\left(x t+\sqrt{1-x^{2}} \sqrt{1-t^{2}}\right)+f\left(x t-\sqrt{1-x^{2}} \sqrt{1-t^{2}}\right)\right], \quad t, x \in I,
$$

and denote

$$
\hat{b}_{n}(x)=\sum_{\jmath=0}^{n} \hat{\beta}_{\jmath, n} \omega_{\jmath} T_{\jmath}(x)
$$

Taking into account that

$$
\left(\tau_{x} T_{\jmath}\right)(t)=T_{\jmath}(x) T_{\jmath}(t)
$$

from (1.1) we see that

$$
\left(\hat{L}_{n} f\right)(x)=\left[f, \tau_{x} \hat{b}_{n}\right]_{n}
$$

and we can say that $\hat{L}_{n}$ is generated by $\hat{b}_{n}$ or that $\left(\hat{b}_{n}\right)$ is the generating sequence for $\left(\hat{L}_{n}\right)$.

However it is clear that $\tau_{x}$ is a positive operator and moreover that for $n \geq 2$

$$
\left(\hat{L}_{n} T_{k}\right)(x)=\hat{\beta}_{k, n} T_{k}(x), \quad k \in \mathbb{N}_{0} .
$$

Further $\hat{\beta}_{k, n}, k=0,1, \cdots, n$, are the Cheby'shev coefficients of $\hat{b}_{n}$. This implies that when $\hat{b}_{n} \geq 0$ on $I$ and

$$
\int_{-1}^{1} \hat{b}_{n}(t) \frac{d t}{\sqrt{1-t^{2}}}=1,
$$


then $\hat{L}_{n}: C^{\prime}(I) \rightarrow \mathrm{II}_{n}$ is a positive linear operator with $\hat{L}_{n} e_{0}=e_{0}, e_{k}(t)=t^{k}$. If we apply $\hat{L}_{n}$ on the inequalities

$$
\begin{gathered}
-1 \leq T_{k}(t) \leq 1, \quad t \in I \\
4\left(1-T_{1}(t)\right)-\left(1-T_{2}(t)\right)=2(t-1)^{2} \geq 0
\end{gathered}
$$

and then select $x=1$ (see (1.4)), one finds

$$
-1 \leq \hat{\beta}_{k, n} \leq 1
$$

and

$$
0 \leq 1-\hat{\beta}_{2, n} \leq 4\left(1-\hat{\beta}_{1, n}\right)
$$

Now

$$
\begin{aligned}
& \left(\hat{L}_{n} e_{1}\right)(x)=e_{1}(x)-x\left(1-\hat{\beta}_{1, n}\right) \\
& \left(\hat{L}_{n} e_{2}\right)(x)=\hat{\beta}_{2, n} e_{2}(x)+\frac{1-\hat{\beta}_{2, n}}{2} .
\end{aligned}
$$

In conclusion, from (1.5) we see that $\lim _{n \rightarrow \infty} \hat{\beta}_{1, n}=1$ is a sufficient condition for the Korovkin conditions

$$
\lim _{n \rightarrow \infty}\left\|e_{k}-\hat{L}_{n} e_{k}\right\|=0, \quad k=1,2 \text {. }
$$

In this present paper we will consider the modified summability methods of $L_{n}$ given by

$$
\left(L_{n}^{\star} f\right)(x):=L_{n}^{\star}\left(z_{0, n}, \cdots, z_{n, n} ; f, x\right)=\sum_{j=0}^{n} \beta_{,, n} \omega,[f, T,]_{n} T,(x),
$$

with the generating polynomial (see (4.3) in [9])

$$
b_{n}(x)=\kappa_{n} \frac{\left(1-x \cos \frac{2 \pi}{n+2}\right)\left(1-T_{n+2}(x)\right)}{(1-x)\left(x-\cos \frac{2 \pi}{n+2}\right)^{2}}=\sum_{j=0}^{n} \beta_{, n} \omega, T,(x)
$$

where $\kappa_{n}=\frac{1}{\pi(n+2)} \sin ^{2} \frac{\pi}{n+2}$ and

$$
\beta_{j, n}=\frac{n-j+2}{n+2} \cos ^{2} \frac{j \pi}{n+2}+\frac{\cos \frac{\pi}{n+2}}{(n+2) \sin \frac{\pi}{n+2}} \cos \frac{j \pi}{n+2} \sin \frac{j \pi}{n+2} .
$$

Without claim of completeness there are in some papers [5],[6],[12] other examples of similar sequences like presented in (1.6).

For this discrete linear positive approximation method in (1.6) we have an instance of algebraic polynomials, for which we can prove (see (2.2)) an estimate of A.F. Timan [13] for all $x \in I$

$$
\left|f(x)-\left(L_{n}^{\star} f\right)(x)\right| \leq C \omega\left(f ; \frac{\sqrt{1-x^{2}}}{n}+\frac{1}{n^{2}}\right), \quad n \in \mathbf{N},
$$

where $\omega\left(f ; \delta_{n}\right):=\sup \left\{|f(t+h)-f(t)| ;|h| \leq \delta_{n}, t, t+h \in I\right\}$ and the constant $C$ is independent of $f$ and $n$.

\section{MAIN RESULTS}

It is the aim of this present note to show that the local order of approximation by means of $\left(L_{n}^{\star}\right)$ is comparable with those furnished by the best approximation polynomials. At the end of this explanations we get a conclusion to prove a problem of P.L. Butzer (see remark 2.4). 
Now let us state our central theorem:

THEOREM 2.1 Let $L^{\star}=\left(L_{n}^{\star}\right), n \in \mathbb{N}$, be defined as in (1.6) and

$$
\Delta_{n}(x):=\frac{\sqrt{1-x^{2}}}{n}+\frac{|x|}{n^{2}}, \quad x \in I .
$$

Then for $f \in C(I), x \in I$,

$$
\left|f(x)-\left(L_{n}^{\star} f\right)(x)\right| \leq 40 \omega\left(f ; \Delta_{n}(x)\right)
$$

and further

$$
\left|f(x)-\left(L_{n}^{\star} f\right)(x)\right| \leq\left(3+2 \pi^{2}\right)\left\{\omega_{2}\left(f ; n^{-1}\right)+\frac{|x|}{n} \omega\left(f ; n^{-1}\right)\right\},
$$

where $\omega_{2}(f ; \delta):=\sup \{|f(x-\delta)-2 f(x)+f(x+\delta)| ; x, x \pm \delta \in I, 0 \leq \delta \leq h\}$ is the second modulus of smoothness of $f$.

PROOF: Our starting point is to construct together with a sequence $A=\left(A_{n}\right)$ of approximation operators - by means of a $\Theta$ - transformation - a new sequence of operators $B=\left(B_{n}\right)=\Theta(A)$.

So, to prove this above estimates, we employ the transformation technique from [9].

Therefore we denote by $X$ the normed linear spaces $C(I)$ or $L_{\omega}^{p}(I), 1 \leq p<\infty$, supplied with norms $\|f\|_{C(I)}=\|f\|:=\max _{t \in I}|f(t)|$ for $f \in C(I)$, respectively $\|f\|_{p}=\left[\int_{-1}^{1}|f(t)|^{p} \omega(t) d t\right]^{\frac{1}{p}}$, where $f$ is an element of the Lebesque space $L_{\omega}^{p}(I)$ with the weight $\omega(t)=\frac{1}{\sqrt{1-t^{2}}}$.

Let us use the well - known convolution product $\star: L_{\omega}^{1}(I) \times L_{\omega}^{1}(I) \rightarrow L_{\omega}^{1}(I)$

$$
(f \star g)(x)=\int_{-1}^{1} f(t)\left(\tau_{x} g\right)(t) \omega(t) d t
$$

According to the Mehler - Hermite quadrature formula, i.e.

$$
\int_{-1}^{1} h(t) \omega(t) d t \approx \frac{\pi}{n+1} \sum_{k=0}^{n} h\left(z_{k, n}\right)
$$

which is exact for algebraic polynomials $h$ of degree $\leq 2 n+1$, we have

$$
[f, g]_{n}=\int_{-1}^{1} f(t) g(t) \omega(t) d t, \quad f g \in \Pi_{2 n+1} .
$$

The polynomial $L_{n}^{\star} f$ can be now expressed as follows

$$
\begin{aligned}
\left(L_{n}^{\star} f\right)(x) & =\sum_{j=0}^{n} \beta_{\jmath, n} \omega_{j}[f, T,]_{n} T,(x)=\frac{\pi}{n+1} \sum_{k=0}^{n} f\left(z_{k, n}\right) \sum_{j=0}^{n} \beta_{\jmath, n} \omega, T,(x) T,\left(z_{k, n}\right) \\
& =\frac{\pi}{n+1} \sum_{k=0}^{n} f\left(z_{k, n}\right)\left(\tau_{x} b_{n}\right)\left(z_{k, n}\right) .
\end{aligned}
$$

If

$$
\left(B_{n} f\right)(x)=\int_{-1}^{1} f(t)\left(\tau_{x} b_{n}\right)(t) \omega(t) d t=\left(f \star b_{n}\right)(x), \quad f \in X,
$$

where the polynomial sequence $b_{n} \in \mathcal{P}^{+}:=\left\{b=\left(b_{n}\right) \mid b_{n}(x) \geq 0, x \in I ; \int_{-1}^{1} b_{n}(t) \omega(t) d t=1\right\}$ is
defined by 


$$
b_{n}(x)=\sum_{j=0}^{n} \beta_{\jmath . n} \omega_{\jmath} T,(x),
$$

then for $f \in \mathrm{II}_{n+1}$ we have

$$
L_{n}^{\star} f=B_{n} f
$$

Since the translation operator $\tau_{x}$ is a positive linear operator, we can see that these properties transfer to the discrete operator $L_{n}^{\star}$ - that means, $L_{n}^{\star}$ is linear and positive.

Now let us describe for our purpose the so called $\Theta$ - transformation [9]:

We start with a polynomial sequence $a=\left(a_{n}\right) \in \mathcal{P}^{1}:=\left\{a=\left(a_{n}\right) \mid a \in \mathcal{P}^{+}\right.$and $\forall n \in \mathbb{N}_{0}$ $\left.\exists z_{0}:=z_{0}(n) \in I: a_{n}\left(z_{0}\right)=a_{n}\left(1, z_{0}\right)=0\right\}$, and the mapping $l: \mathcal{P}^{1} \rightarrow \mathcal{P}^{+}$,

$$
l(a)=b=\left(b_{n}\right)
$$

where

$$
b_{n}(x)=\frac{1}{c_{n}} \frac{a_{n+1}\left(x, \bar{z}_{0}\right)}{1-x}, \quad c_{n}=\int_{-1}^{1} \frac{a_{n+1}\left(t, \bar{z}_{0}\right)}{1-t} \omega(t) d t, \quad \bar{z}_{0}=z_{0}(n+1) .
$$

Then the operator sequence $B=\left(B_{n}\right), B_{n} f=f \star b_{n}$ is called the $\Theta$ - transformation of the sequence $A=\left(A_{n}\right)$, defined by $A_{n} f=f \star a_{n}$, and we write

$$
B=\Theta(A) \text {. }
$$

If we consider the case $a=\left(a_{n}\right), a_{n}(x)=\frac{1-T_{n+1}(x)}{\pi(n+1)(1-x)}$, with $z_{0}=z_{0}(n)=\cos \frac{2 \pi}{n+1}$, then our $\left(B_{n}\right)$ from (2.5) is the $\Theta$ - transformation of Fejér operators

$$
\begin{gathered}
A=\left(F_{n}\right), \\
\left(F_{n} f\right)(x)=\int_{-1}^{1} f(t) \sum_{\jmath=0}^{n}\left(1-\frac{j}{n+1}\right) \omega_{\jmath} T_{\jmath}(x) T_{j}(t) \omega(t) d t, \quad f \in X .
\end{gathered}
$$

Taking into account that $L_{n}^{\star}$ is a discrete form of $B_{n}=\Theta\left(F_{n}\right)$, we conclude, by means of thesrem 4.2 from [9] that

$$
\left|f(x)-\left(L_{n}^{\star} f\right)(x)\right| \leq 4 \omega\left(f ; \epsilon_{n}(x)\right)
$$

with

$$
\epsilon_{n}(x)=\sqrt{1-x^{2}} \sin \frac{\pi}{n+2}+|x| \sin ^{2} \frac{\pi}{n+2} \leq \pi^{2} \Delta_{n}(x) .
$$

At the same time

$$
\left|f(x)-\left(L_{n}^{\star} f\right)(x)\right| \leq\left(3+2 \pi^{2}\right)\left(\omega_{2}\left(f ; n^{-1}\right)+\frac{|x|}{n} \omega\left(f ; n^{-1}\right)\right), \quad \text { (see [9], theorem 4.3), }
$$

which completes our proof.

REMARK 2.2 We get for the convergence multipliers ([9], (3.3))

$$
\begin{aligned}
\beta_{j, n} & =\frac{1}{(n+2) c_{n}}\left(\frac{1-z_{0}-T_{\jmath+1}\left(z_{0}\right)+T_{\jmath}\left(z_{0}\right)}{\left(1-z_{0}\right)^{2}}+\frac{(n+1-j)\left(1+T_{\jmath}\left(z_{0}\right)\right)}{1-z_{0}}\right) \\
& =\frac{2}{(n+2)\left(1-z_{0}\right) c_{n}}\left(\frac{\sin (j+1) \frac{\pi}{n+2} \cos \frac{j \pi}{n+2}}{\sin \frac{\pi}{n+2}}+(n+1-j) \cos ^{2} \frac{j \pi}{n+2}\right)
\end{aligned}
$$




$$
=\frac{2}{\left(1-z_{0}\right) c_{n}}\left(\frac{n-j+2}{n+2} \cos ^{2} \frac{j \pi}{n+2}+\frac{\cos \frac{\pi}{n+2}}{(n+2) \sin \frac{\pi}{n+2}} \sin \frac{j \pi}{n+2} \cos \frac{j \pi}{n+2}\right),
$$

where together with $\beta_{0, n}=\frac{2}{\left(1-z_{0}\right) c_{n}}$

$$
c_{n}=\frac{2}{1-z_{0}}=\frac{1}{1-\beta_{1, n}}=\frac{1}{\sin ^{2} \frac{\pi}{n+2}}
$$

and we find the form in (1.7).

The local behaviour of $f$ is measured by suitable maximal functions taking into consideration the fact that a single local change of $f$ can influence the polynomial operator $L_{n}^{\star}$ as a whole. Therefore the local error $f-L_{n}^{\star} f$ is estimated by the pointwise Lipschitz type maximal function

$$
f_{\alpha} \sim(x):=\sup _{t \neq x, t \in I} \frac{|f(x)-f(t)|}{|t-x|^{\alpha}}
$$

for $f \in L_{1}^{1}(I)$ bounded and $x \in I, \alpha \in(0,1]$, introduced by B. Lenze [7]. For similar results with other operators see [8],[11],[10].

THEOREM 2.3 Let $n \in N, \alpha \in(0,1]$ and $f \in L_{1}^{1}(I)$ be bounded. Then for all $x \in I$

$$
\left|f(x)-\left(L_{n}^{\star} f\right)(x)\right| \leq \pi^{2} f_{\alpha}^{\sim}(x)\left(\Delta_{n}(x)\right)^{\alpha}
$$

PROOF: From the definition of $f_{\alpha}^{\sim}(x)$ and with Hölder inequality for $p=\frac{1}{\alpha} \geq 1$

$$
\begin{aligned}
\left|f(x)-\left(L_{n}^{\star} f\right)(x)\right| & \leq L_{n}^{\star}(|f(x)-f(t)| ; x) \leq f_{\alpha}^{\sim}(x) L_{n}^{\star}\left(|x-t|^{\alpha} ; x\right) \\
& \leq f_{\alpha}^{\sim}(x)\left(L_{n}^{\star}\left(|x-t|^{p \alpha} ; x\right)\right)^{\frac{1}{p}}=f_{\alpha} \sim(x)\left(L_{n}^{\star}(|x-t| ; x)\right)^{\alpha} .
\end{aligned}
$$

With $1-\beta_{1, n}=\sin ^{2} \frac{\pi}{n+2}$ and

we find the following enclosure

$$
\frac{1-\beta_{2, n}}{2}=\frac{2}{n+2} \sin ^{2} \frac{\pi}{n+2}\left(2(n+1) \cos ^{2} \frac{\pi}{n+2}+1\right) \leq \frac{2 \pi^{2}}{(n+2)^{2}},
$$

$$
\begin{aligned}
|x| \sin ^{2} \frac{\pi}{n+2} \leq L_{n}^{\star}(|t-x| ; x) & \leq \frac{\sqrt{2\left(1-x^{2}\right)} \pi}{n+2}+|x| \sin ^{2} \frac{\pi}{n+2} \\
& \leq \pi^{2} \Delta_{n}(x) \quad([9],(2.6)),
\end{aligned}
$$

which proves this theorem.

REMARK 2.4 P.L.Butzer presented on a conference in Budapest [1] the following problem: Can one construct a triangular matrix of distinct nodes $\left\{x_{k, n}\right\}_{k=0}^{n}, n \in \mathbb{N}_{0}, x_{k, n} \in I$, and a triangular matrix of positive fundamental functions $\left\{\varphi_{k, n}\right\}_{k=0}^{n}$, defined on $I$, such that the linear summator operators

are algebraic polynomials of degree $n$, and satisfy

$$
\left(L_{n} f\right)(x):=\sum_{k=0}^{n} f\left(x_{k, n}\right) \varphi_{k, n}(x), \quad f \in C(I),
$$

$$
\left\|L_{n} f-f\right\|_{C(I)}=\mathcal{O}\left(n^{-\alpha}\right),
$$

provided $f \in \operatorname{Lip}_{2}(\alpha, C), 0<\alpha \leq 2$. 
We get a solution of this problem. if we use for $\delta \in(0,1]$ and $f \in L_{\imath} p_{2}(\alpha, C), 0<\alpha \leq 2$, the estimates for $\omega_{2}(f ; \delta) \leq C^{\prime} \delta^{\prime \prime} . C^{\prime}:=$ const. and

$$
\delta_{\omega}(f: \delta) \leq\left\{\begin{array}{cc}
2\|f\| \delta^{\alpha} & , \quad a \in(0,1] \\
\left\|f^{\prime}\right\| \delta^{\alpha} & , \quad a \in(1,2]
\end{array}\right.
$$

We find so with a positive constant $M=M(f)$

$$
\omega_{2}(f ; \delta)+|x| \delta \omega(f ; \delta) \leq M \delta^{\alpha}, \quad a \in(0,2] . \delta \in(0,1], x \in I .
$$

If we choose $\delta=\frac{1}{n}$, from (2.3) we get

$$
\left|f(x)-\left(L_{n}^{\star} f\right)(x)\right| \leq \frac{M}{n^{\alpha}}, \quad x \in I .
$$

Finally the linear summator operators $\left(L_{n}^{\star}\right)$ have the co - domain in $\Pi_{n}$ and satisfy (2.8) provided $f \in L_{\imath} p_{2}(\alpha, C), 0<\alpha \leq 2$, i.e. a solution for the problem proposed by P.L.Butzer.

\section{References}

[1] BUTZER, P.L., Legendre Transform Methods in the Solution of Basic Problems in Algebraic Approximation, in: Functions, Series and Operators Vol.I (J.Szabados and B.Sz.-Nagy, eds.) North - Holland, Amsterdam (1983) 277 - 301.

[2] BUTZER, P.L. AND STENS, R.L., The operational properties of Chebyshev transform (II), Fractional derivatives, Proc.Internat.Conf. on the Theory of Approximation of Functions (Kaluga, 1975), Moscow, (1977) $49-61$.

[3] BUTZER, P.L. AND STENS, R.L., Chebyshev transform methods in the theory of best algebraic approximation, Abh. Math. Sem. Univ. Hamburg, 45, (1976) $165-190$.

[4] BUTZER, P.L., STENS, R.L. AND WEHRENS, M., Approximation by algebraic convolution integrals, Approximation Theory and Functional Analysis (J.B.Prolla, ed.) Math. Studies Vol.35, North - Holland, Amsterdam, (1979), 71 - 120.

[5] CAO, J.D. AND GONSKA, H.H., Approximation by Boolean Sums of Positive Linear Operators III, Estimates for some Numerical Approximation Schemes; Numer. Funct. Anal. and Optimiz., $10(7 \& 8)(1989)$.

[6] CAO, J.D. AND GONSKA, H.H., On Butzer's Problem concerning Approximation by Algebraic Polynomials, Proceedings of the 6 - th South Eastern Approximation Theorists International Conference (Memphis/ 1991) (preprint).

[7] LENZE, B., On Lipschitz - type maximal functions and their smoothness spaces, Proc. Netherl. Acad. Sci. A 91 (1988), 53 - 63 .

[8] LENZE, B., Local error estimates for Bernstein and Kantorovič polynomials via maximal functions, submitted to: Mitteilungen der Mathematischen Gesellschaft in Hamburg .

[9] LUPAŞ, A. AND MACHE, D.H., The Degree of Approximation by a Class of Linear Positive Operators, (1992) (preprint).

[10] MACHE, D.H., Gewichtete Simultanapproximation in der $L_{p}$ - Metrik durch das Verfahren der Kantorovič Operatoren, Dissertation Dortmund (1991).

[11] MACHE, D.H. AND MÜLLER, M.W., New local error estimates for Meyer - König and Zeller Kantorovič - operators using maximal functions, (to appear in Mathematica (Cluj.)).

[12] MILLS, T.M. AND VARMA, A.K., On the Summability of Lagrange Interpolation, J. Appr. Theory 9, (1973) $349-356$.

[13] TIMAN, A.F., Strengthening of Jackson's theorem on best approximation of continuous functions given on a finite interval of the real axis, Dokl. Akad. Nauk SSSR 78 (1951) (Russian), $17-20$. 


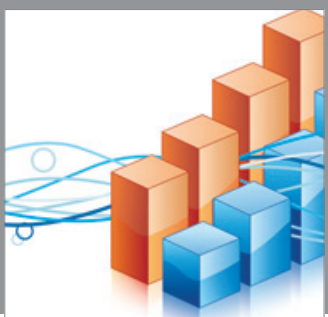

Advances in

Operations Research

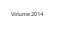

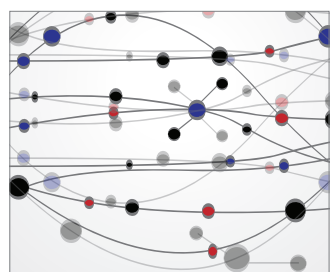

\section{The Scientific} World Journal
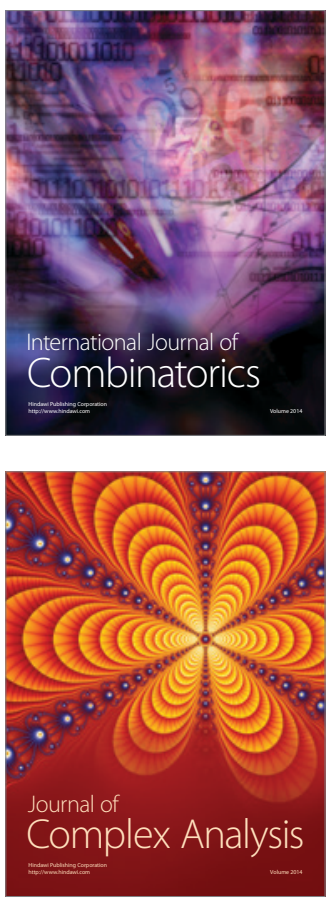

International Journal of

Mathematics and

Mathematical

Sciences
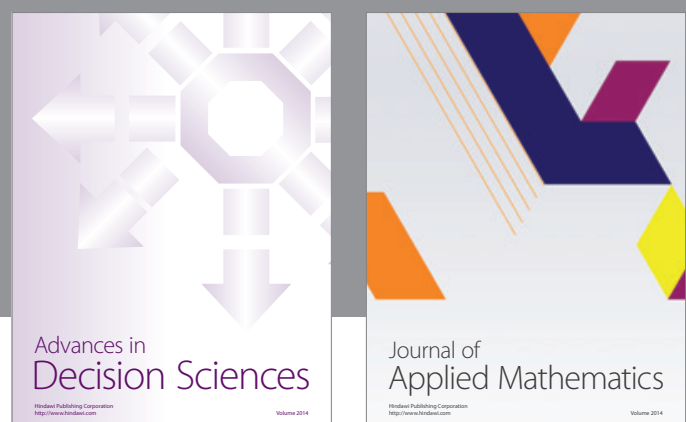

Journal of

Applied Mathematics
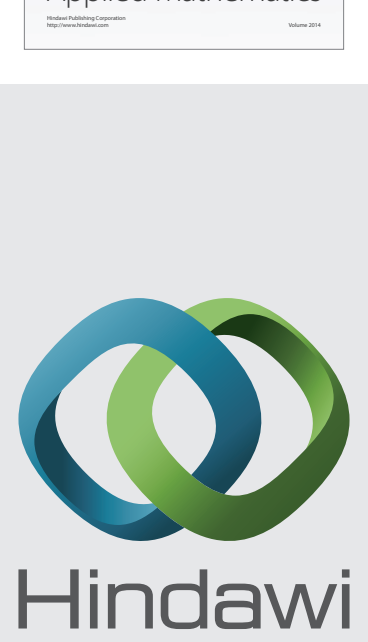

Submit your manuscripts at http://www.hindawi.com
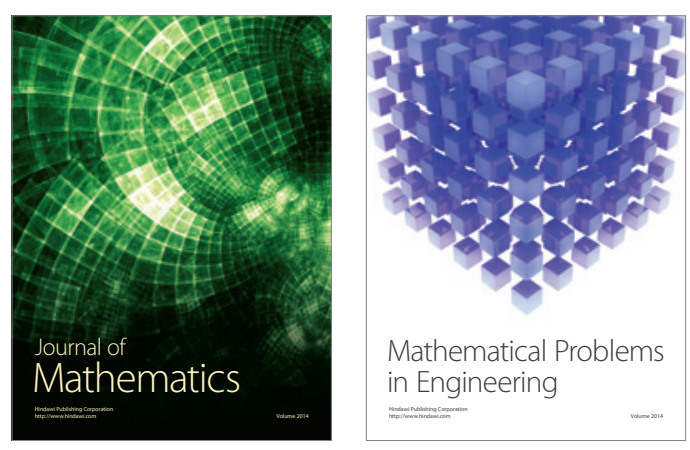

Mathematical Problems in Engineering
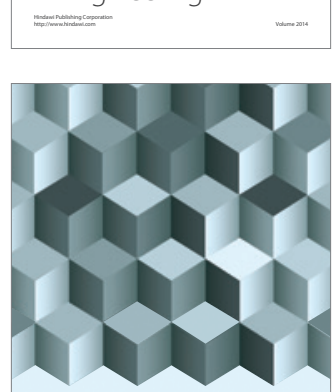

Journal of

Function Spaces
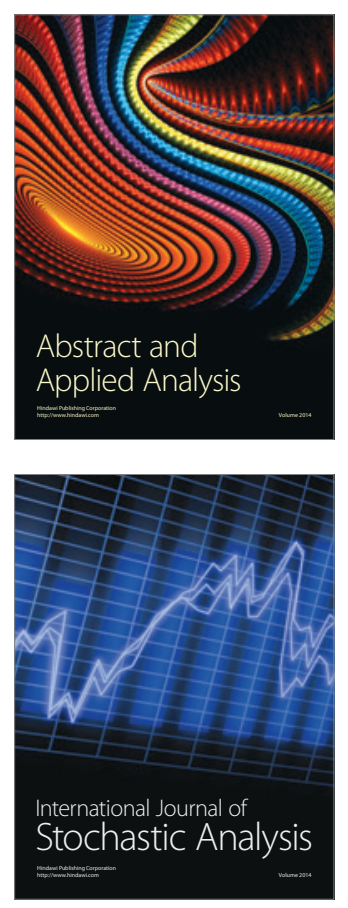

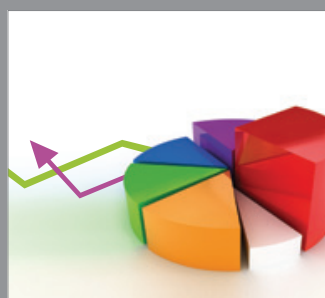

ournal of

Probability and Statistics

Promensencen
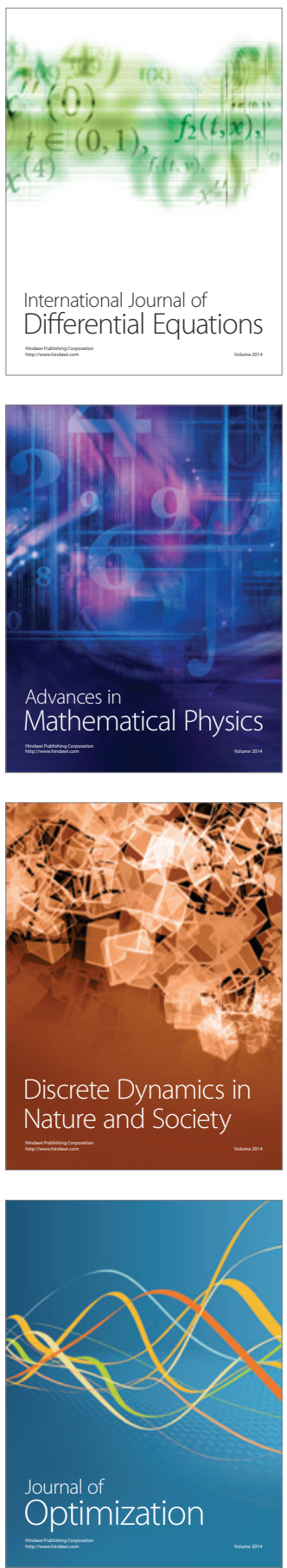